\title{
Experimental
}

Materials: Propargyl bromide, iodine, morpholine, copper(I) iodide, pyrrolidine, propyl bromide, sodium hydride, potassium carbonate, barium oxide, barium hydroxide octahydrate, triethylamine and butylamine were purchased from Fluka (Switzerland). Dibutyltindilaurate, 4-pentyn-1-ol, butylamine, hexyl isocyanate, phenyl isocyanate and triphosgene were purchased from Aldrich (USA). Butyl isocyanate was purchased from Merck (Germany) and used without further purification. tertbutylcalix[4]arene was prepared according to the literature. ${ }^{22}$ Tetrahydrofuran was distilled over sodium and benzophenone, acetonitrile was distilled over calcium hydride and stored over molecular sieves. Other analytical grade solvents were used as received without further distillation. Unless otherwise noted, all reactions were carried out under nitrogen atmosphere. Column chromatography was performed using Merck silica gel 60 (70-230 mesh).

Analytical instruments: ${ }^{1} \mathrm{H}$ and ${ }^{13} \mathrm{C}$ NMR spectra were recorded on Varian Mercury $400 \mathrm{MHz}$ NMR spectrometer (Varian, USA) and AC Bruker 200, 250 and $500 \mathrm{MHz}$ NMR spectrometer (Bruker, USA) using the residual solvent proton resonance of $\mathrm{CDCl}_{3}$ at $7.26 \mathrm{ppm}$ as the reference. Elemental analysis was performed on PE 2400 Series II (Perkin-Elmer, USA). Infrared spectra were measured from KBr pellets on a Nicolet Impact 410 FT-IR spectrophotometer (Thermo Nicolet, USA). UV-Vis spectra were recorded on Varian Cary 100 Bio UV-Visible spectrophotometer (Varian, USA). The melting points were investigated by Mettler Toledo DSC 823e (Mettler Toledo, USA) in aluminium standard cell (40 $\mu \mathrm{L}$ ) as a holder at the heating rate of $10{ }^{\circ} \mathrm{C} / \mathrm{min}$. The FT-Raman experiments were performed by exciting neat solid samples with a $\mathrm{cw} \mathrm{Nd:YAG} \mathrm{laser} \mathrm{at} 1.064 \mu \mathrm{m}$, and the spectra were recorded on the same IR spectrometer with a Raman accessory in a $180^{\circ}$ optical collection geometry. GPC analysis was measured on a Water system (Waters, USA), with a Water 600 pump, a Water 2414 refractive index detector and a set of Styragel ${ }^{\circledR}$ columns (HR1, HR3 and HR4) at $35{ }^{\circ} \mathrm{C}$ using tetrahydrofuran as an eluent at the flow rate of $1.00 \mathrm{~mL} / \mathrm{min}$. 


\section{Synthesis procedures}

\section{5,27-dipropagyloxy-26,28-dihydroxy-tert-butylcalix[4]arene (1): A mixture of potassium}

carbonate $(1.71 \mathrm{~g}, 12.34 \mathrm{mmol})$, tert-butylcalix[4]arene $(4.01 \mathrm{~g}, 6.18 \mathrm{mmol})$ in acetonitrile $(200 \mathrm{~mL})$ was stirred at room temperature for $1 \mathrm{~h}$. A solution of propargyl bromide $(1.24 \mathrm{~g}, 12.98 \mathrm{mmol})$ in acetonitrile $(50 \mathrm{~mL})$ was added dropwise into the stirred mixture over $30 \mathrm{~min}$. The reaction mixture was refluxed for $48 \mathrm{~h}$ and was then allowed to cool to room temperature. The reaction mixture was filtered to remove insoluble particles and the filtrate was concentrated by a rotating evaporator. The concentrated reaction mixture was added with $2 \mathrm{M} \mathrm{HCl}(100 \mathrm{~mL})$ and extracted with dichloromethane $(3 \times 100 \mathrm{~mL})$. The combined organic extract was washed with brine $(100 \mathrm{~mL})$, dried over anhydrous $\mathrm{Na}_{2} \mathrm{SO}_{4}$, filtered and evaporated to dryness in vacuo. The crude mixture was crystallized from $\mathrm{CH}_{2} \mathrm{Cl}_{2} / \mathrm{CH}_{3} \mathrm{OH}$ to afford $\mathbf{1}$ as a white solid $\left(3.72 \mathrm{~g}, 83 \%\right.$ yield). $\mathrm{Mp} 232{ }^{\circ} \mathrm{C}$ (decomposed), IR (KBr, cm $\left.{ }^{-1}\right)$ v 3435, 3280, 2958, 2869, 2121, 1597, 1541, 1482, 1363, 1198, ${ }^{1} \mathrm{H}$ NMR (200 MHz, $\left.\mathrm{CDCl}_{3}\right) \delta(\mathrm{ppm}): 7.13$ (s, 4H, Ar- $\left.\underline{\mathrm{H}}\right)$, $6.78(\mathrm{~s}, 4 \mathrm{H}, \operatorname{Ar}-\underline{\mathrm{H}}), 6.55(\mathrm{~s}, 2 \mathrm{H}, \mathrm{OH}), 4.80\left(\mathrm{~d}, J=4.0 \mathrm{~Hz}, 4 \mathrm{H}, \mathrm{OC}_{2}\right), 4.42(\mathrm{~d}, J=14.0 \mathrm{~Hz}, 4 \mathrm{H}$, $\left.\operatorname{ArC} \underline{H}_{2} \mathrm{Ar}\right), 3.38\left(\mathrm{~d}, J=14.0 \mathrm{~Hz}, 4 \mathrm{H}, \mathrm{ArC}_{2} \mathrm{Ar}\right), 2.58(\mathrm{t}, J=4.0 \mathrm{~Hz}, 2 \mathrm{H}, \mathrm{C} \equiv \mathrm{C} \underline{\mathrm{H}}), 1.35\left(\mathrm{~s}, 18 \mathrm{H},\left(\mathrm{C}_{3}\right)_{3}\right)$, $0.94\left(\mathrm{~s}, 18 \mathrm{H},\left(\mathrm{CH}_{3}\right)_{3}\right)$.

\section{5,26,27-tripropargyloxy-28-hydroxy-tert-butylcalix[4]arene (2): A mixture of $\mathrm{Ba}(\mathrm{OH})_{2} \cdot 8 \mathrm{H}_{2} \mathrm{O}$} (3.66 g, $11.60 \mathrm{mmol}), \mathrm{BaO}(1.02 \mathrm{~g}, 6.63 \mathrm{mmol})$ and tert-butylcalix[4]arene $(2.15 \mathrm{~g}, 3.31 \mathrm{mmol})$ were dissolved in tetrahydrofuran $(50.0 \mathrm{~mL})$ and the solution was stirred at room temperature for $1 \mathrm{~h}$. A solution of propargyl bromide $(4.93 \mathrm{~g}, 33.0 \mathrm{mmol})$ in tetrahydrofuran $(30 \mathrm{~mL})$ was added dropwise into the stirred mixture over $30 \mathrm{~min}$. The reaction mixture was refluxed for $48 \mathrm{~h}$ and was then allowed to cool to room temperature. The reaction mixture was filtered to remove insoluble particles and the filtrate was concentrated by a rotating evaporator. The concentrated reaction mixture was added with $2 \mathrm{M} \mathrm{HCl}$ $(50 \mathrm{~mL})$ and extracted with dichloromethane $(3 \times 60 \mathrm{~mL})$. The combined organic extract was dried over anhydrous $\mathrm{Na}_{2} \mathrm{SO}_{4}$, filtered and the solvent was removed to give a brown oil residue. The residue was purified by column chromatography with dichloromethane/hexane (15/85) to give 2 as a white solid 
$(67 \%$ yield, $1.69 \mathrm{~g}) . \mathrm{Mp} 157{ }^{\circ} \mathrm{C}, \mathrm{IR}\left(\mathrm{KBr}, \mathrm{cm}^{-1}\right) \vee 3458,3291,2959,2868,2122,2011,1733,1598$, 1480, 1363, 1197, ${ }^{1} \mathrm{H}$ NMR (200 MHz, $\left.\mathrm{CDCl}_{3}\right) \delta(\mathrm{ppm}): 7.18$ (s, 2H, Ar- $\left.\underline{\mathrm{H}}\right), 7.13$ (s, 2H, Ar- $\left.\underline{\mathrm{H}}\right), 6.63$ (s, $2 \mathrm{H}, \operatorname{Ar}-\underline{\mathrm{H}}), 6.59$ (s, 2H, Ar- $\underline{\mathrm{H}}), 5.83(\mathrm{~s}, 1 \mathrm{H}, \mathrm{OH}), 5.08\left(\mathrm{~d}, J=2.5 \mathrm{~Hz}, 2 \mathrm{H}, \mathrm{OC}_{2}\right), 4.70(\mathrm{~d}, J=3.0 \mathrm{~Hz}$, $\left.4 \mathrm{H}, \mathrm{OC}_{2}\right), 4.66\left(\mathrm{~d}, J=14.0 \mathrm{~Hz}, 2 \mathrm{H}, \mathrm{ArC}_{2} \mathrm{Ar}\right), 4.43\left(\mathrm{~d}, J=14.0 \mathrm{~Hz}, 2 \mathrm{H}, \mathrm{ArC}_{2} \mathrm{Ar}\right), 3.36(\mathrm{~d}, J=14.0$ $\left.\mathrm{Hz}, 2 \mathrm{H}, \mathrm{ArC}_{2} \mathrm{Ar}\right), 3.27\left(\mathrm{~d}, J=14.0 \mathrm{~Hz}, 2 \mathrm{H}, \mathrm{ArC}_{2} \mathrm{Ar}\right), 2.56(\mathrm{t}, J=3.0 \mathrm{~Hz}, 2 \mathrm{H}, \mathrm{C} \equiv \mathrm{C} \underline{\mathrm{H}}), 2.50(\mathrm{t}, J=$ $2.5 \mathrm{~Hz}, 1 \mathrm{H}, \mathrm{C} \equiv \mathrm{C} \underline{\mathrm{H}}), 1.37\left(\mathrm{~s}, 18 \mathrm{H},\left(\mathrm{C}_{3}\right)_{3}\right), 0.90\left(\mathrm{~s}, 9 \mathrm{H},\left(\mathrm{C}_{3}\right)_{3}\right), 0.89$ (s, 9H, $\left.\left(\mathrm{C}_{3}\right)_{3}\right)$.

25,26,27,28-tetra(propargyloxy)-tert-butylcalix[4]arene (3): A mixture of potassium carbonate (43.37 g, $313.76 \mathrm{mmol})$, tert-butylcalix[4]arene $(10.18 \mathrm{~g}, 15.68 \mathrm{mmol})$ in acetonitrile $(200 \mathrm{~mL}) \mathrm{was}$ stirred at room temperature for $1 \mathrm{~h}$. A solution of propargyl bromide $(23.33 \mathrm{~g}, 156.88 \mathrm{mmol})$ in acetonitrile $(50 \mathrm{~mL})$ was added dropwise into the stirred mixture over $30 \mathrm{~min}$. The reaction mixture was allowed to reflux for $48 \mathrm{~h}$ and then allowed to cool to room temperature. The reaction mixture was filtered to remove insoluble particles and the filtrate was concentrated by a rotating evaporator. The concentrated reaction mixture was added with $2 \mathrm{M} \mathrm{HCl}(100 \mathrm{~mL})$ and extracted with dichloromethane $(3 \times 100 \mathrm{~mL})$. The combined organic extract was washed with brine $(100 \mathrm{~mL})$, dried over anhydrous $\mathrm{Na}_{2} \mathrm{SO}_{4}$, filtered and evaporated to dryness in vacuo. The crude mixture was crystallized from $\mathrm{CH}_{2} \mathrm{Cl}_{2} / \mathrm{CH}_{3} \mathrm{OH}$ to afford 3 as a white solid (10.68 g, 85\% yield). Mp $107{ }^{\circ} \mathrm{C}$, IR $\left(\mathrm{KBr}, \mathrm{cm}^{-1}\right) \vee 3310$, 3285, 2960, 2866, 2121, 1602, 1478, 1196, 1021, 1018, ${ }^{1} \mathrm{H}$ NMR (400 MHz, $\left.\mathrm{CDCl}_{3}\right) \delta(\mathrm{ppm}): 6.78$ (s, $8 \mathrm{H}, \operatorname{Ar}-\underline{\mathrm{H}}), 4.80\left(\mathrm{~d}, J=2.4 \mathrm{~Hz}, 8 \mathrm{H}, \mathrm{OC}_{2}\right), 4.60\left(\mathrm{~d}, J=13.2 \mathrm{~Hz}, 4 \mathrm{H}, \mathrm{ArCH}_{2} \mathrm{Ar}\right), 3.16(\mathrm{~d}, J=13.2 \mathrm{~Hz}$, $\left.4 \mathrm{H}, \operatorname{ArC} \underline{H}_{2} \mathrm{Ar}\right), 2.48(\mathrm{t}, J=2.4 \mathrm{~Hz}, 3 \mathrm{H}, \mathrm{C} \equiv \mathrm{C} \underline{\mathrm{H}}), 1.07\left(\mathrm{~s}, 36 \mathrm{H},\left(\mathrm{C}_{3}\right)_{3}\right)$.

\section{5,27-dipropargyloxy-26,28-dipropyl-tert-butylcalix[4]arene (4): 1 (1.03 g, $1.42 \mathrm{mmol})$ and $\mathrm{NaH}$}

$(0.40 \mathrm{~g}, 14.16 \mathrm{mmol})$ which was washed by hexane $(3 \times 10 \mathrm{~mL})$ were dissolved in tetrahydrofuran $(20$ $\mathrm{mL}$ ), and the solution was stirred at room temperature for $1 \mathrm{~h}$. Then the solution of propyl bromide (1.74 $\mathrm{g}, 14.16 \mathrm{mmol})$ in tetrahydrofuran $(20 \mathrm{~mL})$ was added by dropwise. After adding was complete, the reaction mixture was allowed to reflux for $24 \mathrm{~h}$. The reaction mixture was then allowed to cool to room temperature and quenched by ethanol $(20 \mathrm{~mL})$. The solvent was removed in vacuo to obtain yellow 
residue and then the residue was extracted with dichloromethane $(3 \times 30 \mathrm{~mL})$. The combined organic layers were washed with brine $(50 \mathrm{~mL})$, dried over anhydrous $\mathrm{Na}_{2} \mathrm{SO}_{4}$, filtered and removed solvent in vacuo to give brown oil residue. The residue was isolated by column chromatography with dichloromethane as an eluent to afford 4 as a white solid $(0.35 \mathrm{~g}, 31 \%$ yield $) . \mathrm{Mp} 190{ }^{\circ} \mathrm{C}$, IR $\left(\mathrm{KBr}, \mathrm{cm}^{-1}\right)$ v 3310, 2960, 2872, 2124, 1602, 1478, 1198, 1120, 1016, ${ }^{1} \mathrm{H}$ NMR (400 MHz, $\left.\mathrm{CDCl}_{3}\right) \delta(\mathrm{ppm}): 7.09$ (s, 4H, Ar- $\underline{\mathrm{H}}), 6.45$ (s, 4H, Ar- $\underline{\mathrm{H}}), 5.02\left(\mathrm{~s}, J=2.4 \mathrm{~Hz}, 4 \mathrm{H}, \mathrm{OC}_{2} \mathrm{Ar}\right), 4.52\left(\mathrm{~d}, J=12.0 \mathrm{~Hz}, 4 \mathrm{H}, \operatorname{ArC} \underline{\mathrm{H}}_{2} \mathrm{Ar}\right)$, $3.71\left(\mathrm{t}, J=6.0 \mathrm{~Hz}, 4 \mathrm{H}, \underline{\mathrm{CH}}_{2} \mathrm{CH}_{2} \mathrm{CH}_{3}\right), 3.14\left(\mathrm{~d}, J=12.0 \mathrm{~Hz}, 4 \mathrm{H}, \mathrm{ArC}_{2} \mathrm{Ar}\right), 2.39(\mathrm{t}, J=2.4 \mathrm{~Hz}, 3 \mathrm{H}$, $\mathrm{C} \equiv \mathrm{C} \underline{\mathrm{H}}), 2.00\left(\mathrm{~m}, 4 \mathrm{H}, \mathrm{CH}_{2} \underline{\mathrm{C}}_{2} \mathrm{CH}_{3}\right), 1.33\left(\mathrm{~s}, 18 \mathrm{H},\left(\mathrm{C}_{3}\right)_{3}\right), 1.05\left(\mathrm{t}, J=6.0 \mathrm{~Hz}, 6 \mathrm{H}, \mathrm{CH}_{2} \mathrm{CH}_{2} \mathrm{C}_{3}\right), 0.83$ $\left(\mathrm{s}, 18 \mathrm{H},\left(\mathrm{CH}_{3}\right)_{3}\right)$.

25,26,27-tripropargyloxy-28-propyl-tert-butylcalix[4]arene (5): $2(0.93 \mathrm{~g}, 1.22 \mathrm{mmol})$ and $\mathrm{NaH}$

$(0.15 \mathrm{~g}, 6.08 \mathrm{mmol})$ which was washed by hexane $(3 \times 10 \mathrm{~mL})$ were dissolved in tetrahydrofuran $(20$ $\mathrm{mL}$ ), and the solution was stirred at room temperature for $1 \mathrm{~h}$. Then the solution of propyl bromide $(0.75 \mathrm{~g}, 6.08 \mathrm{mmol})$ in tetrahydrofuran $(20 \mathrm{~mL})$ was added by dropwise. After adding was complete, the reaction mixture was allowed to reflux for $24 \mathrm{~h}$. The reaction mixture was then allowed to cool to room temperature and quenched by ethanol $(20 \mathrm{~mL})$. The solvent was removed in vacuo to obtain yellow residue and then the residue was extracted with dichloromethane $(3 \times 30 \mathrm{~mL})$. The combined organic layers were washed with brine $(50 \mathrm{~mL})$, dried over anhydrous $\mathrm{Na}_{2} \mathrm{SO}_{4}$, filtered and removed solvent in vacuo to give brown oil residue. The residue was isolated by column chromatography with dichloromethane as an eluent to afford $\mathbf{5}$ as a white solid $(0.80 \mathrm{~g}, 82 \%$ yield $) . \mathrm{Mp} 140{ }^{\circ} \mathrm{C}, \mathrm{IR}\left(\mathrm{KBr}, \mathrm{cm}^{-1}\right)$ $v$ 3309, 3287, 2960, 2869, 2120, 2007, 1599, 1478, 1363, 1300, 1197, $1016{ }^{1} \mathrm{H}$ NMR (400 MHz,

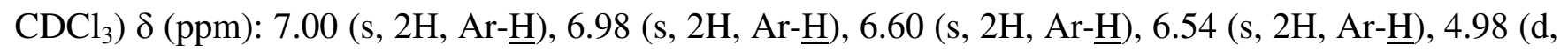
$\left.J=2.4 \mathrm{~Hz}, 2 \mathrm{H}, \mathrm{CH}_{2} \mathrm{C} \equiv \mathrm{C}\right), 4.89\left(\mathrm{~d}, J=2.4 \mathrm{~Hz}, 2 \mathrm{H}, \mathrm{CH}_{2} \mathrm{C} \equiv \mathrm{C}\right), 4.63\left(\mathrm{~d}, J=2.0 \mathrm{~Hz}, 2 \mathrm{H}, \mathrm{CH}_{2} \mathrm{C} \equiv \mathrm{C}\right), 4.59$ $\left(\mathrm{d}, J=12.5 \mathrm{~Hz}, 2 \mathrm{H}, \operatorname{ArC} \underline{\mathrm{H}}_{2} \mathrm{Ar}\right), 4.51\left(\mathrm{~d}, J=12.5 \mathrm{~Hz}, 2 \mathrm{H}, \operatorname{ArC}_{2} \mathrm{Ar}\right), 3.76(\mathrm{t}, J=7.4 \mathrm{~Hz}, 2 \mathrm{H}$, $\left.\mathrm{C}_{2} \mathrm{CH}_{2} \mathrm{CH}_{3}\right), 3.17\left(\mathrm{~d}, J=5.6 \mathrm{~Hz}, 2 \mathrm{H}, \mathrm{ArC}_{2} \mathrm{Ar}\right), 3.13\left(\mathrm{~d}, J=5.6 \mathrm{~Hz}, 2 \mathrm{H}, \mathrm{ArCH}_{2} \mathrm{Ar}\right), 2.4(\mathrm{t}, J=2.0 \mathrm{~Hz}$ 
$1 \mathrm{H}, \mathrm{C} \equiv \mathrm{C} \underline{\mathrm{H}}), 2.44(\mathrm{t}, J=2.4 \mathrm{~Hz}, 2 \mathrm{H}, \mathrm{C} \equiv \mathrm{C} \underline{\mathrm{H}}), 2.00\left(\mathrm{~m}, 2 \mathrm{H}, \mathrm{CH}_{2} \underline{\mathrm{C}}_{2} \mathrm{CH}_{3}\right), 1.28\left(\mathrm{~s}, 18 \mathrm{H},\left(\mathrm{C}_{3}\right)_{3}\right), 1.07(\mathrm{t}$, $\left.J=7.4 \mathrm{~Hz}, 3 \mathrm{H}, \mathrm{CH}_{2} \mathrm{CH}_{2} \mathrm{C}_{3}\right), 0.96\left(\mathrm{~s}, 9 \mathrm{H},\left(\mathrm{C}_{3}\right)_{3}\right), 0.94\left(\mathrm{~s}, 9 \mathrm{H},\left(\mathrm{C}_{3}\right)_{3}\right)$.

5-iodo-4-pentynol: A solution of morpholine $(33.25 \mathrm{~mL}, 381.60 \mathrm{mmol})$ in toluene $(350 \mathrm{~mL})$ at $45^{\circ} \mathrm{C}$ was treated with iodine $(13.56 \mathrm{~g}, 53.42 \mathrm{mmol})$, shielded from light and stirred for $1 \mathrm{~h}$. A solution of 4pentyn-1-ol (3.21 g, $38.16 \mathrm{mmol})$ in toluene $(30 \mathrm{~mL})$ was added and the reaction was stirred at $45^{\circ} \mathrm{C}$ for $1 \mathrm{~h}$. The reaction mixture was cooled at room temperature and filtered to remove salt. The filtrate was poured over diethyl ether $(200 \mathrm{~mL})$ and saturated aqueous $\mathrm{Na}_{2} \mathrm{~S}_{2} \mathrm{O}_{3}$ solution $(100 \mathrm{~mL})$ and shaken vigorously until the organic layer was colorless. The organic layers were separated, washed with $\mathrm{Na}_{2} \mathrm{~S}_{2} \mathrm{O}_{3}(100 \mathrm{~mL})$, dried over anhydrous $\mathrm{Na}_{2} \mathrm{SO}_{4}$, filtered, concentrated and purified by column chromatography (ethyl acetate/hexane $=7 / 93)$ to afford 5-iodo-4-pentynol as a light yellow oil (6.97 g, 87\% yield). ${ }^{1} \mathrm{H}$ NMR (400 MHz, $\left.\mathrm{CDCl}_{3}\right) \delta(\mathrm{ppm}): 3.78\left(\mathrm{t}, J=6.0 \mathrm{~Hz}, 2 \mathrm{H}, \mathrm{CH}_{2} \mathrm{OH}\right), 2.53(\mathrm{t}, J=6.0 \mathrm{~Hz}$, $2 \mathrm{H}, \mathrm{C} \equiv \mathrm{CC}_{2}$ ), 1.80 (qui, $J=6.0 \mathrm{~Hz}, 2 \mathrm{H}, \mathrm{CH}_{2} \underline{\mathrm{CH}}_{2} \mathrm{CH}_{2} \mathrm{OH}$ ), 1.61 (bs, $1 \mathrm{H}, \mathrm{OH}$ ).

\section{5,27-di(octa-4,6-diyn-1-ol)oxy-26,28-dipropyl-tert-butylcalix[4]arene (C2A): To a stirred solution}

of $4(0.22 \mathrm{~g}, 0.27 \mathrm{mmol})$, and 5-iodo-4-pentynol $(0.28 \mathrm{~g}, 1.34 \mathrm{mmol})$ in pyrrolidine $(1.0 \mathrm{~mL})$ was added copper(I) iodide $(0.01 \mathrm{~g}, 0.05 \mathrm{mmol})$. After stirring at room temperature for $2 \mathrm{~h}$, the mixture was hydrolyzed with saturated aqueous solution of ammonium chloride $(30 \mathrm{~mL})$ and extracted with diethyl ether $(3 \times 50 \mathrm{~mL})$. The organic layer was dried over anhydrous $\mathrm{Na}_{2} \mathrm{SO}_{4}$, filtered and the solvent was removed in vacuo. The crude product was purified by column chromatography (dichloromethane/ethyl acetate $=10 / 90)$ to obtain $\mathbf{C 2 A}$ as a light yellow solid $(0.10 \mathrm{~g}, 38 \%$ yield $) . \mathrm{Mp} 168^{\circ} \mathrm{C}, \mathrm{IR}\left(\mathrm{KBr}, \mathrm{cm}^{-1}\right) v$ 3414, 2959, 2867, 2253, 1604, 1478, 1362, 1197, 1120, 1011, ${ }^{1} \mathrm{H}$ NMR (400 MHz, $\left.\mathrm{CDCl}_{3}\right) \delta(\mathrm{ppm})$ : 7.09 (s, 4H, Ar- $\underline{\mathrm{H}}), 6.43$ (s, 4H, Ar- $\underline{\mathrm{H}}), 5.01\left(\mathrm{~s}, 4 \mathrm{H}, \mathrm{OC}_{2} \mathrm{C} \equiv \mathrm{C}\right), 4.48\left(\mathrm{~d}, J=13.6 \mathrm{~Hz}, 4 \mathrm{H}, \mathrm{ArC}_{2} \mathrm{Ar}\right)$, $3.76\left(\mathrm{t}, J=6.0 \mathrm{~Hz}, 4 \mathrm{H}, \mathrm{C}_{2} \mathrm{CH}_{2} \mathrm{CH}_{3}\right), 3.71\left(\mathrm{t}, J=7.5 \mathrm{~Hz}, 4 \mathrm{H}, \underline{\mathrm{C}}_{2} \mathrm{OH}\right), 3.14(\mathrm{~d}, J=13.6 \mathrm{~Hz}, 4 \mathrm{H}$, $\operatorname{ArC} \underline{H}_{2} \mathrm{Ar}$ ), 2.43 (t, $J=7.5 \mathrm{~Hz}, 4 \mathrm{H}, \underline{\mathrm{CH}}_{2} \mathrm{CH}_{2} \mathrm{CH}_{2} \mathrm{OH}$ ), $2.00\left(\mathrm{~m}, 4 \mathrm{H}, \mathrm{CH}_{2} \mathrm{C}_{2} \mathrm{CH}_{3}\right.$ ), 1.81 (qui, $J=7.5 \mathrm{~Hz}$, $\left.4 \mathrm{H}, \mathrm{CH}_{2} \mathrm{CH}_{2} \mathrm{OH}\right), 1.33\left(\mathrm{~s}, 18 \mathrm{H},\left(\mathrm{C}_{3}\right)_{3}\right), 1.05\left(\mathrm{t}, J=6.0 \mathrm{~Hz}, 6 \mathrm{H}, \mathrm{CH}_{2} \mathrm{CH}_{2} \mathrm{CH}_{3}\right), 0.82\left(\mathrm{~s}, 18 \mathrm{H},\left(\mathrm{CH}_{3}\right)_{3}\right)$. 
25,26,27-tri(octa-4,6-diyn-1-ol)oxy-28-propyl-tert-butylcalix[4]arene (C3A): To a stirred solution

of $5(0.24 \mathrm{~g}, 0.30 \mathrm{mmol})$, and 5-iodo-4-pentynol $(0.36 \mathrm{~g}, 1.79 \mathrm{mmol})$ in pyrrolidine $(2.00 \mathrm{~mL})$ was added copper(I) iodide $(0.02 \mathrm{~g}, 0.08 \mathrm{mmol})$. After stirring at room temperature for $2 \mathrm{~h}$, the mixture was hydrolyzed with saturated aqueous solution of ammonium chloride $(30 \mathrm{~mL})$ and extracted with diethyl ether $(3 \times 50 \mathrm{~mL})$. The organic layer was dried over anhydrous $\mathrm{Na}_{2} \mathrm{SO}_{4}$, filtered and the solvent was removed in vacuo. The crude product was purified by column chromatography (dichloromethane/ethyl acetate $=50 / 50)$ to give $\mathbf{C 3 A}$ as a light yellow solid $(0.18 \mathrm{~g}, 58 \%$ yield $) . \mathrm{Mp} 145^{\circ} \mathrm{C}, \mathrm{IR}\left(\mathrm{KBr}, \mathrm{cm}^{-1}\right) v$ 3379, 2958, 2867, 2254, 1602, 1478, 1361, 1196, 1119, 1053, $1009{ }^{1} \mathrm{H}$ NMR (400 MHz, $\left.\mathrm{CDCl}_{3}\right) \delta$ (ppm): 7.00 (s, 4H, Ar- $\underline{\mathrm{H}}), 6.58(\mathrm{~s}, 2 \mathrm{H}, \mathrm{Ar}-\underline{\mathrm{H}}), 6.51(\mathrm{~s}, 2 \mathrm{H}, \mathrm{Ar}-\underline{\mathrm{H}}), 5.01\left(\mathrm{~s}, 2 \mathrm{H}, \mathrm{C}_{2} \mathrm{C} \equiv \mathrm{C}\right), 4.87$ (s, 2H, $\left.\mathrm{C}_{2} \mathrm{C} \equiv \mathrm{C}\right), 4.71\left(\mathrm{~s}, 2 \mathrm{H}, \underline{\mathrm{C}}_{2} \mathrm{C} \equiv \mathrm{C}\right), 4.55\left(\mathrm{~d}, J=12.5 \mathrm{~Hz}, 2 \mathrm{H}, \operatorname{ArC} \underline{\mathrm{H}}_{2} \mathrm{Ar}\right), 4.46(\mathrm{~d}, J=12.5 \mathrm{~Hz}, 2 \mathrm{H}$, $\left.\operatorname{ArC} \underline{H}_{2} \mathrm{Ar}\right), 3.76\left(\mathrm{~m}, 8 \mathrm{H}, \underline{\mathrm{CH}}_{2} \mathrm{CH}_{2} \mathrm{CH}_{3}\right.$, and $\left.\underline{\mathrm{CH}}_{2} \mathrm{OH}\right), 3.17$ (d, $\left.J=6.4 \mathrm{~Hz}, 2 \mathrm{H}, \mathrm{ArC}_{2} \mathrm{Ar}\right), 3.14$ (d, $J=6.4$ $\left.\mathrm{Hz}, 2 \mathrm{H}, \mathrm{ArC} \underline{\mathrm{H}}_{2} \mathrm{Ar}\right), 2.44\left(\mathrm{t}, J=6.6 \mathrm{~Hz}, 6 \mathrm{H}, \mathrm{C}_{2} \mathrm{CH}_{2} \mathrm{CH}_{2} \mathrm{OH}\right), 2.00\left(\mathrm{~m}, 4 \mathrm{H}, \mathrm{CH}_{2} \underline{\mathrm{C}}_{2} \mathrm{CH}_{3}\right), 1.81(\mathrm{~m}, 6 \mathrm{H}$, $\left.\mathrm{C}_{2} \mathrm{CH}_{2} \mathrm{OH}\right), 1.25\left(\mathrm{~s}, 18 \mathrm{H},\left(\mathrm{C}_{3}\right)_{3}\right), 1.08\left(\mathrm{t}, J=7.4 \mathrm{~Hz}, 3 \mathrm{H}, \mathrm{CH}_{2} \mathrm{CH}_{2} \mathrm{CH}_{3}\right), 0.92\left(\mathrm{~s}, 9 \mathrm{H},\left(\mathrm{CH}_{3}\right)_{3}\right), 0.87(\mathrm{~s}$ $\left.9 \mathrm{H},\left(\mathrm{C}_{3}\right)_{3}\right)$.

25,26,27,28-tetra(octa-4,6-diyn-1-ol)oxy-tert-butylcalix[4]arene (C4A): To a stirred solution of 3 (4.18 g, $5.22 \mathrm{mmol})$, and 5-iodo-4-pentynol $(10.96 \mathrm{~g}, 52.18 \mathrm{mmol})$ in pyrrolidine $(40.00 \mathrm{~mL})$ was added copper(I) iodide $(0.40 \mathrm{~g}, 2.09 \mathrm{mmol})$. After stirring at room temperature for $2 \mathrm{~h}$, the mixture was hydrolyzed with saturated aqueous solution of ammonium chloride (200 mL) and extracted with diethyl ether $(3 \times 150 \mathrm{~mL})$. The organic layer was dried over anhydrous $\mathrm{Na}_{2} \mathrm{SO}_{4}$, filtered and the solvent was removed in vacuo. The crude product was purified by column chromatography (ethyl acetate) to afford C4A as a light yellow solid (2.73 g, 51\% yield). $\mathrm{Mp} 212^{\circ} \mathrm{C}$ (decomposed), IR (KBr, $\left.\mathrm{cm}^{-1}\right) \vee 3386,2965$, 2905, 2868, 2254, 1688, 1603, 1480, 1362, 1196, ${ }^{1} \mathrm{H}$ NMR (400 MHz, $\left.\mathrm{CDCl}_{3}\right) \delta(\mathrm{ppm}): 6.77$ (s, 8H, Ar$\underline{\mathrm{H}}), 4.82\left(\mathrm{~s}, 8 \mathrm{H}, \mathrm{OC}_{2}\right), 4.53\left(\mathrm{~d}, J=13.2 \mathrm{~Hz}, 4 \mathrm{H}, \mathrm{ArC}_{2} \mathrm{Ar}\right), 3.77\left(\mathrm{t}, J=7.0 \mathrm{~Hz}, 8 \mathrm{H}, \mathrm{C}_{2} \mathrm{OH}\right), 3.17(\mathrm{~d}, J$ $\left.=13.2 \mathrm{~Hz}, 4 \mathrm{H}, \mathrm{ArC}_{2} \mathrm{Ar}\right), 2.45\left(\mathrm{t}, J=7.0 \mathrm{~Hz}, 8 \mathrm{H}, \mathrm{C} \equiv \mathrm{CC}_{\mathrm{H}_{2}}\right), 1.90(\mathrm{bs}, 1 \mathrm{H}, \mathrm{OH}), 1.81$ (qui, $J=7.0 \mathrm{~Hz}$ 8H, $\left.\mathrm{CH}_{2} \underline{\mathrm{C}}_{2} \mathrm{CH}_{2}\right), 1.07\left(\mathrm{~s}, 36 \mathrm{H},\left(\mathrm{CH}_{3}\right)_{3}\right),{ }^{13} \mathrm{C} \mathrm{NMR}\left(\mathrm{CDCl}_{3}\right) \delta(\mathrm{ppm}): 152.5\left(\underline{\mathrm{C}}_{\mathrm{ppso}}-\mathrm{Ar}\right), 145.6\left(\underline{\mathrm{C}}_{\mathrm{para}}-\mathrm{Ar}\right)$ 
$134.1\left(\underline{\mathrm{C}}_{\text {ortho }}-\mathrm{Ar}\right), 125.0\left(\underline{\mathrm{C}}_{\text {meta }}-\mathrm{Ar}\right), 79.9(\mathrm{C} \equiv \underline{\mathrm{C}} \equiv \mathrm{C}), 73.7(\underline{\mathrm{C}} \equiv \mathrm{CC} \equiv \mathrm{C}), 71.0(\mathrm{C} \equiv \mathrm{C} \underline{\mathrm{C}} \equiv \mathrm{C}), 65.6(\mathrm{C} \equiv \mathrm{CC} \equiv \underline{\mathrm{C}})$, $61.9\left(\underline{\mathrm{C}} \mathrm{H}_{2} \mathrm{OH}\right), 61.2\left(\mathrm{OCH}_{2}\right), 33.9\left(\underline{\mathrm{C}}\left(\mathrm{CH}_{3}\right)_{3}\right), 32.2\left(\mathrm{ArCH}_{2} \mathrm{Ar}\right), 31.3\left(\left(\underline{\mathrm{CH}}_{3}\right)_{3}\right), 30.8\left(\mathrm{C} \equiv \mathrm{CCH}_{2} \underline{\mathrm{CH}_{2}}\right), 15.9$ $\left(\mathrm{C} \equiv \mathrm{CCH}_{2}\right)$.

\section{Urethane formation of C2BU, and C3BU}

General procedure for synthesis

Butylamine was dissolved in chloroform. Triphosgene was gradually added to this solution with stirring. Triethylamine was added dropwise to this stirred mixture (a water bath may be needed to keep the temperature below $30^{\circ} \mathrm{C}$ ). The reaction mixture was refluxed for $2 \mathrm{~h}$ before diacetylenic alcohol-tertbutylcalix[4]arene derivatives $[\mathrm{C} 2 \mathrm{~A}$, or $\mathrm{C} 3 \mathrm{~A}]$ and dibutyltindilaurate were added. The stirring was continued for $10 \mathrm{~h}$ at room temperature. The solvent was evaporated, and the residue was redissolved in hexane. The resulting solution was filtered, and the solid was washed several times with hexane. The filtrate was collected, and the solvent was evaporated. The residue was eluted through a silica gel column.

\section{5,27-di[octa-4,6-diyn-1-ol-(carboxy-butyl-urethane)]oxy-26,28-dipropyl-tert-butylcalix[4]arene}

(C2BU): According to urethane formation above, C2BU was synthesized from C2A $(0.07 \mathrm{~g}, 0.07$ mmol), butylamine (0.32 g, $4.32 \mathrm{mmol})$, triphosgene $(0.43 \mathrm{~g}, 1.44 \mathrm{mmol})$, triethylamine $(1.20 \mathrm{~mL}, 8.63$ $\mathrm{mmol})$, and dibutyltindilaurate $(0.18 \mathrm{~g}, 0.29 \mathrm{mmol})$ in chloroform $(10 \mathrm{~mL})$ and purified by column chromatography (ethyl acetate/dichloromethane $=1 / 99)$ to give $\mathbf{C 2 B U}$ as a light yellow solid $(0.05 \mathrm{~g}$, $55 \%$ yield). Mp $100^{\circ} \mathrm{C}, \mathrm{IR}\left(\mathrm{KBr}, \mathrm{cm}^{-1}\right) \vee 3348,2959,2870,2254,1702,1523,1473,1362,1244,1198$,

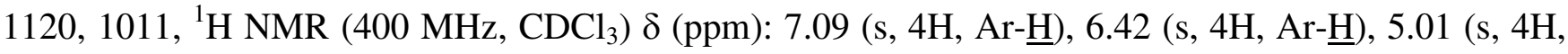
$\left.\mathrm{OC} \underline{\mathrm{H}}_{2} \mathrm{C} \equiv \mathrm{C}\right), 4.69$ (bs, $\left.2 \mathrm{H}, \mathrm{NH}\right), 4.48\left(\mathrm{~d}, J=13.6 \mathrm{~Hz}, 4 \mathrm{H}, \mathrm{ArC} \underline{\mathrm{H}}_{2} \mathrm{Ar}\right), 4.12\left(\mathrm{t}, J=5.8 \mathrm{~Hz}, 4 \mathrm{H}, \mathrm{OCOC}_{2}\right)$, $3.70\left(\mathrm{t}, J=6.0 \mathrm{~Hz}, 4 \mathrm{H}, \underline{\mathrm{C}}_{2} \mathrm{CH}_{2} \mathrm{CH}_{3}\right), 3.14\left(\mathrm{~m}, J=13.6 \mathrm{~Hz}, 8 \mathrm{H}, \mathrm{ArC}_{2} \underline{\mathrm{Ar}}_{2}\right.$ and $\left.\mathrm{NC}_{2}\right), 2.38(\mathrm{t}, J=6.6$ $\mathrm{Hz}, 4 \mathrm{H}, \mathrm{C} \equiv \mathrm{CC}_{2}$ ), 2.00 (m, 4H, $\left.\mathrm{CH}_{2} \mathrm{C}_{2} \mathrm{CH}_{3}\right), 1.84$ (qui, $\left.J=5.8 \mathrm{~Hz}, 4 \mathrm{H}, \mathrm{C} \equiv \mathrm{CCH}_{2} \mathrm{C}_{2}\right), 1.47$ (m, 4H, $\left.\mathrm{CH}_{2} \underline{\mathrm{C}}_{2} \mathrm{CH}_{2} \mathrm{CH}_{3}\right), 1.32\left(\mathrm{~s}, 18 \mathrm{H},\left(\mathrm{C}_{3}\right)_{3}\right), 1.05\left(\mathrm{t}, J=6.0 \mathrm{~Hz}, 6 \mathrm{H}, \mathrm{CH}_{2} \mathrm{CH}_{2} \underline{\mathrm{C}}_{3}\right), 0.92(\mathrm{~m}, 6 \mathrm{H}$, 
$\left.\mathrm{CH}_{2} \mathrm{CH}_{2} \mathrm{CH}_{2} \underline{\mathrm{CH}}_{3}\right), 0.81\left(\mathrm{~s}, 18 \mathrm{H},\left(\mathrm{C}_{3}\right)_{3}\right),{ }^{13} \mathrm{C} \mathrm{NMR}\left(\mathrm{CDCl}_{3}\right) \delta(\mathrm{ppm}): 156.3$ (으), $153.0\left(\underline{\mathrm{C}}_{\mathrm{ipso}}-\mathrm{Ar}\right)$, $152.6\left(\underline{\mathrm{C}}_{\mathrm{ipso}}-\mathrm{Ar}\right), 145.8$ ( $\left.\underline{\mathrm{C}}_{\mathrm{para}}-\mathrm{Ar}\right), 144.0\left(\underline{\mathrm{C}}_{\mathrm{para}}-\mathrm{Ar}\right), 136.3$ ( $\left.\underline{\mathrm{C}}_{\text {ortho }}-\mathrm{Ar}\right), 131.8\left(\underline{\mathrm{C}}_{\text {ortho }}-\mathrm{Ar}\right), 125.4\left(\underline{\mathrm{C}}_{\mathrm{meta}}-\mathrm{Ar}\right)$, $124.3\left(\underline{\mathrm{C}}_{\text {meta }}-\mathrm{Ar}\right), \quad 78.9 \quad\left(\mathrm{C} \equiv \mathrm{CC} \equiv \underline{\mathrm{CCH}}_{2} \mathrm{CH}_{2}\right), \quad 77.4 \quad\left(\mathrm{O}_{\underline{C}}{ }_{2}\right), \quad 74.6 \quad\left(\underline{\mathrm{C}} \equiv \mathrm{CC} \equiv \mathrm{CCH}_{2} \mathrm{CH}_{2}\right), \quad 70.9$

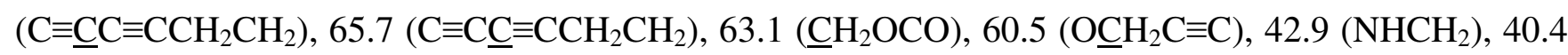
$\left.\left.\left(\mathrm{ArCH}_{2} \mathrm{Ar}\right), 34.1\left(\underline{\mathrm{C}}\left(\mathrm{CH}_{3}\right)_{3}\right), 33.5\left(\underline{\mathrm{C}}\left(\mathrm{CH}_{3}\right)_{3}\right), 31.7\left(\mathrm{NHCH}_{2} \underline{\mathrm{CH}}\right)_{2}\right), 31.7\left(\mathrm{C} \underline{\mathrm{CH}}_{3}\right)_{3}\right), 31.1\left(\mathrm{C}(\underline{\mathrm{CH}})_{3}\right), 28.0$ $\left.\left(\mathrm{CH}_{2} \underline{\mathrm{CH}}_{2} \mathrm{CH}_{2} \mathrm{O}\right), 23.6\left(\underline{\mathrm{CH}}_{2} \mathrm{CH}_{3}\right), 20.1\left(\underline{\mathrm{CH}}_{2} \mathrm{CH}_{3}\right), 16.2\left(\mathrm{C} \equiv \mathrm{C}_{\mathrm{CH}}\right)_{2}\right), 13.8\left(\underline{\mathrm{C}} \mathrm{H}_{3}\right), 10.8\left(\underline{\mathrm{CH}}_{3}\right)$. Anal. Calcd for $\mathrm{C}_{76} \mathrm{H}_{102} \mathrm{~N}_{2} \mathrm{O}_{8}$ : C, 77.91; H, 8.77; N, 2.89. Found: C, 76.28; H, 8.43; N, 3.19.

\section{5,26,27-tri[octa-4,6-diyn-1-ol-(carboxy-butyl-urethane)]oxy-28-propyl-tert-butylcalix[4]arene}

(C3BU): According to urethane formation above, C3BU was synthesized from C3A (0.07 g, 0.07 mmol), butylamine $(0.07 \mathrm{~g}, 1.00 \mathrm{mmol})$, triphosgene $(0.10 \mathrm{~g}, 0.30 \mathrm{mmol})$, triethylamine $(0.28 \mathrm{~mL}, 2.00$ $\mathrm{mmol})$, and dibutyltindilaurate $(0.13 \mathrm{~g}, 0.21 \mathrm{mmol})$ in chloroform $(10 \mathrm{~mL})$ and purified by column chromatography (ethyl acetate/hexane $=15 / 85)$ to give $\mathbf{C 3 B U}$ as a light yellow solid (0.03 g, $32 \%$ yield). $\operatorname{Mp} 117^{\circ} \mathrm{C}, \mathrm{IR}\left(\mathrm{KBr}, \mathrm{cm}^{-1}\right) \vee 3352,2959,2870,2249,1713,1531,1475,1363,1251,1197,1121,1011$, ${ }^{1} \mathrm{H}$ NMR (400 MHz, $\mathrm{CDCl}_{3}$ ) $\delta(\mathrm{ppm}): 7.00$ (s, 4H, Ar- $\left.\underline{\mathrm{H}}\right), 6.56$ (s, 2H, Ar- $\left.\underline{\mathrm{H}}\right), 6.50$ (s, 2H, Ar- $\underline{\mathrm{H}}$ ), 4.99 $\left(\mathrm{s}, 2 \mathrm{H}, \mathrm{OC}_{2} \mathrm{C} \equiv \mathrm{C}\right), 4.88\left(\mathrm{~s}, 2 \mathrm{H}, \mathrm{OC}_{2} \mathrm{C} \equiv \mathrm{C}\right), 4.80(\mathrm{bs}, 3 \mathrm{H}, \mathrm{NH}), 4.70\left(\mathrm{~s}, 2 \mathrm{H}, \mathrm{OC}_{2} \mathrm{C} \equiv \mathrm{C}\right), 4.54(\mathrm{~d}, J=$ $\left.13.0 \mathrm{~Hz}, 2 \mathrm{H}, \mathrm{ArC}_{2} \mathrm{Ar}\right), 4.47\left(\mathrm{~d}, J=13.0 \mathrm{~Hz}, 2 \mathrm{H}, \mathrm{ArC}_{2} \mathrm{Ar}\right), 4.12\left(\mathrm{t}, J=5.6 \mathrm{~Hz}, 6 \mathrm{H}, \underline{\mathrm{C}}_{2} \mathrm{OCO}\right), 3.74$ (t, $\left.2 \mathrm{H}, \underline{\mathrm{C}}_{2} \mathrm{CH}_{2} \mathrm{CH}_{3}\right), 3.15$ (m, 10H, $\mathrm{ArC}_{2} \mathrm{Ar}$ and $\mathrm{NHCH}_{2}$ ), 2.39 (t, J=6.4 Hz, 6H, $\underline{\mathrm{C}}_{2} \mathrm{CH}_{2} \mathrm{CH}_{2} \mathrm{OCO}$ ), $2.03\left(\mathrm{~m}, 4 \mathrm{H}, \mathrm{CH}_{2} \underline{\mathrm{C}}_{2} \mathrm{CH}_{3}\right), 1.86\left(\mathrm{~m}, 6 \mathrm{H}, \mathrm{C}_{2} \mathrm{CH}_{2} \mathrm{OCO}\right), 1.47\left(\mathrm{~m}, 6 \mathrm{H}, \mathrm{CH}_{2} \underline{\mathrm{C}}_{2} \mathrm{CH}_{2} \mathrm{CH}_{3}\right), 1.34(\mathrm{~m}, 6 \mathrm{H}$, $\left.\mathrm{CH}_{2} \mathrm{CH}_{2} \underline{\mathrm{C}}_{2} \mathrm{CH}_{3}\right), 1.25$ (s, 18H, $\left.\left(\mathrm{C}_{3}\right)_{3}\right), 1.07$ (m, 9H, $\left.\mathrm{CH}_{2} \mathrm{CH}_{2} \mathrm{CH}_{2} \mathrm{C}_{3}\right), 0.91$ (s, 9H, $\left.\left(\mathrm{C}_{3}\right)_{3}\right), 0.86$ (s, 9H, $\left.\left(\mathrm{CH}_{3}\right)_{3}\right),{ }^{13} \mathrm{C}$ NMR $\left(\mathrm{CDCl}_{3}\right) \delta(\mathrm{ppm}): 156.5$ (므), $153.3\left(\underline{\mathrm{C}}_{\mathrm{ipso}}-\mathrm{Ar}\right), 152.8\left(\underline{\mathrm{C}}_{\mathrm{ipso}}-\mathrm{Ar}\right), 152.0\left(\underline{\mathrm{C}}_{\mathrm{p} p s o}-\mathrm{Ar}\right)$,

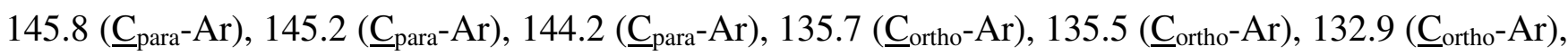

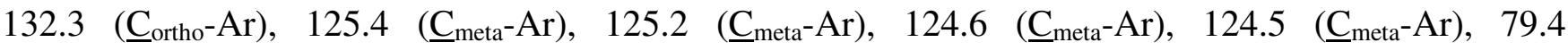
$\left(\mathrm{OCH}_{2} \mathrm{C} \equiv \mathrm{CC} \equiv \underline{\mathrm{C}}\right), \quad 79.2 \quad\left(\mathrm{OCH}_{2} \mathrm{C} \equiv \mathrm{CC} \equiv \underline{\mathrm{C}}\right), \quad 77.4 \quad\left(\underline{\mathrm{CH}}_{2} \mathrm{CH}_{2} \mathrm{CH}_{3}\right), \quad 74.4 \quad\left(\mathrm{OCH}_{2} \underline{\mathrm{C}} \equiv \mathrm{CC} \equiv \mathrm{C}\right), \quad 73.3$ $\left(\mathrm{OCH}_{2} \underline{\mathrm{C}} \equiv \mathrm{CC} \equiv \mathrm{C}\right), \quad 71.2 \quad\left(\mathrm{OCH}_{2} \mathrm{C} \equiv \underline{\mathrm{CC}} \equiv \mathrm{C}\right), \quad 70.8 \quad\left(\mathrm{OCH}_{2} \mathrm{C} \equiv \underline{\mathrm{CC}} \equiv \mathrm{C}\right), \quad 65.7 \quad\left(\mathrm{OCH}_{2} \mathrm{C} \equiv \mathrm{CC} \equiv \mathrm{C}\right), \quad 65.6$ $\left(\mathrm{OCH}_{2} \mathrm{C} \equiv \mathrm{C} \underline{\mathrm{C}} \equiv \mathrm{C}\right), 63.2\left(\underline{\mathrm{CH}}_{2} \mathrm{OCO}\right), 62.4\left(\mathrm{ArOC}_{2}\right), 61.1\left(\mathrm{ArO}_{\underline{C}} H_{2}\right), 40.7\left(\mathrm{NHCH}_{2}\right), 34.1\left(\underline{\mathrm{C}}\left(\mathrm{CH}_{3}\right)_{3}\right)$, 
$33.7\left(\underline{\mathrm{C}}\left(\mathrm{CH}_{3}\right)_{3}\right), 33.6\left(\underline{\mathrm{C}}\left(\mathrm{CH}_{3}\right)_{3}\right), 32.4\left(\mathrm{ArCH}_{2} \mathrm{Ar}\right), 30.0\left(\mathrm{NHCH}_{2} \underline{\mathrm{C}} \mathrm{H}_{2}\right), 31.7\left(\mathrm{ArCH}_{2} \mathrm{Ar}\right), 31.6\left(\mathrm{C}(\underline{\mathrm{CH}})_{3}\right)$,

$31.2\left(\mathrm{C}\left(\underline{\mathrm{CH}}_{3}\right)_{3}\right), 31.1\left(\mathrm{C}\left(\underline{\mathrm{CH}}_{3}\right)_{3}\right), 29.7\left(\mathrm{C} \equiv \mathrm{CCH}_{2} \underline{\mathrm{CH}_{2}}\right), 28.0\left(\mathrm{C} \equiv \mathrm{CCH}_{2} \underline{\mathrm{CH}_{2}}\right), 27.9\left(\mathrm{CH}_{2} \underline{\mathrm{CH}}_{2} \mathrm{CH}_{3}\right), 23.7$

$\left(\underline{\mathrm{CH}}_{2} \mathrm{CH}_{3}\right), 19.9\left(\mathrm{C} \equiv \mathrm{CCLH}_{2} \mathrm{CH}_{2}\right), 16.3\left(\mathrm{C} \equiv \mathrm{CCH}_{2} \mathrm{CH}_{2}\right), 13.7 \quad\left(\underline{\mathrm{CH}}_{3}\right), 10.4 \quad\left(\underline{\mathrm{CH}}_{3}\right)$. Anal. Calcd for $\mathrm{C}_{86} \mathrm{H}_{113} \mathrm{~N}_{3} \mathrm{O}_{10}$ : C, 76.58; H, 8.44; N, 3.12. Found: C, 74.40; H, 7.71; N 3.21.

\section{Urethane formation of C4BU, C4HU and C4PU}

General procedure for synthesis

Diacetylenic alcohol-tert-butylcalix[4]arene derivatives and dibutyltindilaurate were dissolved in tetrahydrofuran, $\mathrm{R}-\mathrm{N}=\mathrm{C}=\mathrm{O}$ (commercial available) was added dropwise in this solution with stirring at 0 ${ }^{\circ} \mathrm{C}$. The mixture was allowed to warm to room temperature and the stirring was continued for $2 \mathrm{~h}$ before ice-water was added. Tetrahydrofuran was evaporated and the aqueous residue was extracted with $\mathrm{CH}_{2} \mathrm{Cl}_{2}$ several times and brine. The combined organic layers were dried over anhydrous $\mathrm{Na}_{2} \mathrm{SO}_{4}$, filtered and the solvent was evaporated in vасио. The crude product was eluted through a silica gel column.

\section{5,26,27,28-tetra[octa-4,6-diyn-1-ol-(carboxy-butyl-urethane)]oxy-tert-butylcalix[4]arene}

(C4BU): According to urethane formation above, C4BU was synthesized from C4A (0.85 g, 0.75 mmol), butyl isocyanate $(0.75 \mathrm{~g}, 7.53 \mathrm{mmol})$, and dibutyltindilaurate $(1.90 \mathrm{~g}, 3.01 \mathrm{mmol})$ in tetrahydrofuran $(20 \mathrm{~mL})$ and purified by column chromatography (ethyl acetate/hexane $=45 / 55)$ to give

C4BU as a white solid (0.96 g, 84\% yield). Mp $119^{\circ} \mathrm{C}, \mathrm{IR}\left(\mathrm{KBr}, \mathrm{cm}^{-1}\right)$ v 3332, 2956, 2930, 2872, 2261, 1690, 1543, 1481, 1362, 1273, 1199, 1155, 1122, ${ }^{1} \mathrm{H}$ NMR (400 MHz, $\left.\mathrm{CDCl}_{3}\right) \delta$ (ppm): 6.77 (s, 8H, Ar$\underline{\mathrm{H}}), 4.82\left(\mathrm{~s}, 8 \mathrm{H}, \mathrm{OC}_{2}\right.$ and $\left.\mathrm{NH}\right), 4.53\left(\mathrm{~d}, J=13.2 \mathrm{~Hz}, 4 \mathrm{H}, \operatorname{ArC}_{2} \mathrm{Ar}\right), 4.13(\mathrm{t}, J=5.8 \mathrm{~Hz}, 8 \mathrm{H}$, $\left.\mathrm{OCOC}_{2}\right), 3.17\left(\mathrm{~m}, 12 \mathrm{H}, \operatorname{ArCH}_{2} \mathrm{Ar}\right.$ and $\left.\mathrm{NC}_{2}\right), 2.39\left(\mathrm{t}, J=6.8 \mathrm{~Hz}, 8 \mathrm{H}, \mathrm{C} \equiv \mathrm{CC}_{2} \underline{C H}_{2}\right), 1.87(\mathrm{qui}, J=$ $6.0 \mathrm{~Hz}, 8 \mathrm{H}, \mathrm{C} \equiv \mathrm{CCH}_{2} \underline{\mathrm{CH}}_{2}$ ), 1.47 (qui, $J=6.6 \mathrm{~Hz}, 8 \mathrm{H}, \mathrm{CH}_{2} \underline{\mathrm{C}}_{2} \mathrm{CH}_{2} \mathrm{CH}_{3}$ ), $1.33\left(\mathrm{~m}, 8 \mathrm{H}, \mathrm{CH}_{2} \mathrm{CH}_{3}\right.$ ), 1.06 (s, 36H $\left.\left(\mathrm{CH}_{3}\right)_{3}\right), 0.95\left(\mathrm{t}, J=7.4 \mathrm{~Hz}, 12 \mathrm{H}, \underline{\mathrm{CH}}_{3}\right),{ }^{13} \mathrm{C} \mathrm{NMR}\left(\mathrm{CDCl}_{3}\right) \delta(\mathrm{ppm}): 156.5(\underline{\mathrm{CO}}), 152.7\left(\underline{\mathrm{C}}_{\mathrm{pps}^{-}}\right.$ Ar), $145.5\left(\underline{\mathrm{C}}_{\mathrm{para}}-\mathrm{Ar}\right), 134.1$ ( $\left.\underline{\mathrm{C}}_{\text {ortho }}-\mathrm{Ar}\right), 125.0\left(\underline{\mathrm{C}}_{\text {meta }}-\mathrm{Ar}\right), 79.4(\mathrm{C} \equiv \underline{\mathrm{CC}} \equiv \mathrm{C}), 73.9(\underline{\mathrm{C}} \equiv \mathrm{CC} \equiv \mathrm{C}), 71.0$ 
$(\mathrm{C} \equiv \mathrm{C} \underline{\mathrm{C}} \equiv \mathrm{C}), 65.6(\mathrm{C} \equiv \mathrm{CC} \equiv \underline{\mathrm{C}}), 63.2\left(\underline{\mathrm{C}} \mathrm{H}_{2} \mathrm{OCO}\right), 61.9\left(\mathrm{O}^{\mathrm{CH}} \mathrm{H}_{2}\right), 40.7\left(\mathrm{NH}^{\mathrm{C}} \mathrm{H}_{2}\right), 33.9\left(\underline{\mathrm{C}}\left(\mathrm{CH}_{3}\right)_{3}\right), 32.3$

(ArC $\left.\left.\underline{H}_{2} \mathrm{Ar}\right), 32.0\left(\mathrm{NHCH}_{2} \underline{\mathrm{CH}}\right)_{2}\right), 31.3\left(\left(\underline{\mathrm{CH}}_{3}\right)_{3}\right), 28.0\left(\mathrm{C} \equiv \mathrm{C}^{\mathrm{C}} \mathrm{H}_{2}\right), 19.9\left(\mathrm{CH}_{2} \underline{\mathrm{CH}_{2}} \mathrm{CH}_{2}\right), 16.3\left(\underline{\mathrm{CH}}_{2} \mathrm{CH}_{3}\right)$, 13.7 $\left(\mathrm{CH}_{3}\right)$. Anal. Calcd for $\mathrm{C}_{96} \mathrm{H}_{124} \mathrm{~N}_{4} \mathrm{O}_{12}: \mathrm{C}, 75.56 ; \mathrm{H}, 8.19 ; \mathrm{N}, 3.67$. Found: $\mathrm{C}, 75.58 ; \mathrm{H}, 8.39 ; \mathrm{N} 3.65$.

\section{5,26,27,28-tetra[octa-4,6-diyn-1-ol-(carboxy-hexyl-urethane)]oxy-tert-butylcalix[4]arene}

(C4HU): C4HU was prepared as described above from C4A (0.54 g, $0.48 \mathrm{mmol})$, butyl isocyanate $(0.61$ $\mathrm{g}, 4.78 \mathrm{mmol})$, and dibutyltindilaurate $(1.21 \mathrm{~g}, 1.91 \mathrm{mmol})$ in tetrahydrofuran $(15 \mathrm{~mL})$ and purified by column chromatography (ethyl acetate/hexane $=27 / 73)$ to give $\mathbf{C} 4 \mathbf{H U}$ as a white solid $(0.35 \mathrm{~g}, 45 \%$ yield). Mp $103^{\circ} \mathrm{C}, \mathrm{IR}\left(\mathrm{KBr}, \mathrm{cm}^{-1}\right) \vee$ 3331, 2958, 2931, 2859, 2258, 1691, 1618, 1544, 1480, 1362, 1254 , 1197, 1122, ${ }^{1} \mathrm{H}$ NMR (400 MHz, $\left.\mathrm{CDCl}_{3}\right) \delta$ (ppm): 6.76 (s, 8H, Ar-프), 4.91 (bs, NH), 4.80 (s, 8H, $\left.\mathrm{OC}_{2}\right), 4.53\left(\mathrm{~d}, J=12.8 \mathrm{~Hz}, 4 \mathrm{H}, \operatorname{ArC} \underline{\mathrm{H}}_{2} \mathrm{Ar}\right), 4.12\left(\mathrm{t}, J=6.0 \mathrm{~Hz}, 8 \mathrm{H}, \underline{\mathrm{C}}_{2} \mathrm{OCO}\right), 3.16(\mathrm{~d}, J=12.8 \mathrm{~Hz}$, $4 \mathrm{H}, \mathrm{ArC}_{2} \mathrm{Ar}$ ), $3.12\left(\mathrm{t}, J=6.0 \mathrm{~Hz}, 8 \mathrm{H}, \mathrm{NHC}_{2}\right), 2.38\left(\mathrm{t}, J=6.4 \mathrm{~Hz}, 8 \mathrm{H}, \mathrm{C} \equiv \mathrm{CC}_{2}\right.$ ), 1.85 (qui, $J=6.4$ $\left.\mathrm{Hz}, 8 \mathrm{H}, \mathrm{C} \equiv \mathrm{CCH}_{2} \underline{\mathrm{C}}_{2}\right), 1.47\left(\mathrm{~m}, 8 \mathrm{H}, \mathrm{NHCH}_{2} \underline{\mathrm{C}}_{2}\right), 1.27$ (m, 24H, $\left.\underline{\mathrm{C}}_{2} \underline{\mathrm{C}}_{2} \underline{\mathrm{C}}_{2} \mathrm{CH}_{3}\right), 1.05$ (s, 36H, $\left.\left(\mathrm{C}_{3}\right)_{3}\right), 0.87\left(\mathrm{t}, J=6.2 \mathrm{~Hz}, 12 \mathrm{H}, \underline{\mathrm{C}}_{3}\right) .{ }^{13} \mathrm{C} \mathrm{NMR}\left(\mathrm{CDCl}_{3}\right) \delta(\mathrm{ppm}): 156.4(\underline{\mathrm{CO}}), 152.5\left(\underline{\mathrm{C}}_{\mathrm{pps} 0}-\mathrm{Ar}\right), 145.5$ ( $\left.\underline{\mathrm{C}}_{\text {para }}-\mathrm{Ar}\right), 134.0$ ( $\left.\underline{\mathrm{C}}_{\text {ortho }}-\mathrm{Ar}\right), 125.0$ ( $\left.\underline{\mathrm{C}}_{\text {meta }}-\mathrm{Ar}\right), 79.3(\mathrm{C} \equiv \underline{\mathrm{C}} \equiv \mathrm{C}), 73.8(\underline{\mathrm{C}} \equiv \mathrm{CC} \equiv \mathrm{C}), 71.0(\mathrm{C} \equiv \mathrm{C} \underline{\mathrm{C}} \equiv \mathrm{C}), 65.6$ $(\mathrm{C} \equiv \mathrm{CC} \equiv \underline{\mathrm{C}}), 63.1\left(\underline{\mathrm{CH}}_{2} \mathrm{OCO}\right), 61.8\left(\mathrm{O}_{\underline{C H}}\right), 40.9\left(\mathrm{NH}_{2} \mathrm{H}_{2}\right), 33.8\left(\underline{\mathrm{C}}\left(\mathrm{CH}_{3}\right)_{3}\right), 32.2\left(\mathrm{ArCH}_{2} \mathrm{Ar}\right), 31.4$ $\left(\underline{\mathrm{CH}_{2}} \mathrm{CH}_{2} \mathrm{CH}_{3}\right), 31.2\left(\left(\underline{\mathrm{CH}}_{3}\right)_{3}\right), 29.8\left(\mathrm{NHCH}_{2} \underline{\mathrm{CH}_{2}}\right), 27.9\left(\mathrm{C} \equiv \mathrm{CCH}_{2} \underline{\mathrm{C}} \mathrm{H}_{2}\right), 26.3\left(\mathrm{NHCH}_{2} \mathrm{CH}_{2} \underline{\mathrm{CH}_{2}}\right), 22.5$ $\left(\underline{\mathrm{CH}}_{2} \mathrm{CH}_{3}\right), 16.2\left(\underline{\mathrm{CH}}_{3}\right), 13.9\left(\mathrm{C} \equiv \mathrm{C}_{\mathrm{CH}}\right)$. Anal. Calcd for $\mathrm{C}_{104} \mathrm{H}_{140} \mathrm{~N}_{4} \mathrm{O}_{12}: \mathrm{C}, 76.25 ; \mathrm{H}, 8.61 ; \mathrm{N}, 3.42$. Found: C, 76.24; H, 8.64; N 3.76 .

\section{5,26,27,28-tetra[octa-4,6-diyn-1-ol-(carboxy-phenyl-urethane)]oxy-tert-butylcalix[4]arene}

(C4PU): C4PU was prepared as described above from C4A $(0.36 \mathrm{~g}, 0.32 \mathrm{mmol})$, phenyl isocyanate $(0.38 \mathrm{~g}, 3.19 \mathrm{mmol})$, and dibutyltindilaurate $(0.81 \mathrm{~g}, 1.27 \mathrm{mmol})$ in tetrahydrofuran $(15 \mathrm{~mL})$ and purified by column chromatography (ethyl acetate/hexane $=22 / 78)$ to give $\mathbf{C 4 P U}$ as a light yellow solid $(0.33 \mathrm{~g}$, $65 \%$ yield $) . \mathrm{Mp} 107{ }^{\circ} \mathrm{C}, \mathrm{IR}\left(\mathrm{KBr}, \mathrm{cm}^{-1}\right) \vee 3317,2959,2252,1714,1602,1541,1480,1445,1315,{ }^{1} \mathrm{H}$ NMR (400 MHz, $\left.\mathrm{CDCl}_{3}\right) \delta(\mathrm{ppm}): 7.37\left(\mathrm{~d}, J=8.0 \mathrm{~Hz}, 8 \mathrm{H}, \underline{\mathrm{H}}_{\text {ortho }}-\mathrm{Ar}\right), 7.28$ (t, $J=8.0 \mathrm{~Hz}, 8 \mathrm{H}, \underline{\mathrm{H}}_{\text {meta- }}$-Ar), $7.04\left(\mathrm{t}, J=8.0 \mathrm{~Hz}, 4 \mathrm{H}, \underline{\mathrm{H}}_{\text {para }}-\mathrm{Ar}\right), 6.90(\mathrm{bs}, \mathrm{NH}), 6.78\left(\mathrm{~s}, 8 \mathrm{H}, \underline{\mathrm{H}}-\mathrm{Ar}_{\text {calix }}\right), 4.82\left(\mathrm{~s}, 8 \mathrm{H}, \mathrm{OC}_{2}\right), 4.54(\mathrm{~d}, J=$ 
$\left.12.8 \mathrm{~Hz}, 4 \mathrm{H}, \mathrm{ArC}_{2} \mathrm{Ar}\right), 4.27\left(\mathrm{t}, J=6.0 \mathrm{~Hz}, 8 \mathrm{H}, \mathrm{OCOC}_{2}\right), 3.18\left(\mathrm{~d}, J=12.8 \mathrm{~Hz}, 4 \mathrm{H}, \mathrm{ArC}_{2} \underline{\mathrm{H}}_{2} \mathrm{Ar}\right), 2.44$ $\left(\mathrm{t}, J=7.0 \mathrm{~Hz}, 8 \mathrm{H}, \mathrm{C} \equiv \mathrm{CC}_{2}\right), 1.96\left(\mathrm{~m}, 8 \mathrm{H}, \mathrm{CH}_{2} \mathrm{CH}_{2} \mathrm{CH}_{2}\right), 1.06\left(\mathrm{~s}, 36 \mathrm{H},\left(\mathrm{C}_{3}\right)_{3}\right) .{ }^{13} \mathrm{C} \mathrm{NMR}\left(\mathrm{CDCl}_{3}\right)$ $\delta(\mathrm{ppm}): 158.3(\underline{\mathrm{CO}}), 152.6\left(\underline{\mathrm{C}}_{\mathrm{ppso}}-\mathrm{Ar}_{\text {calix }}\right), 145.6\left(\underline{\mathrm{C}}_{\mathrm{para}}-\mathrm{Ar}_{\text {calix }}\right), 138.5\left(\underline{\mathrm{C}}_{\mathrm{ppso}}-\mathrm{Ar}\right), 134.1\left(\underline{\mathrm{C}}_{\text {ortho }}-\mathrm{Ar}_{\text {calix }}\right)$, 129.0 ( $\left.\underline{\mathrm{C}}_{\text {ortho- }}-\mathrm{Ar}\right), 125.0\left(\underline{\mathrm{C}}_{\text {meta }}-\mathrm{Ar}_{\text {calix }}\right), 123.4\left(\underline{\mathrm{C}}_{\text {meta }}-\mathrm{Ar}\right), 118.7\left(\underline{\mathrm{C}}_{\mathrm{para}}-\mathrm{Ar}\right), 79.2(\mathrm{C} \equiv \underline{\mathrm{CC}} \equiv \mathrm{C}), 74.0$ $(\underline{\mathrm{C}} \equiv \mathrm{CC} \equiv \mathrm{C}), 73.4(\mathrm{C} \equiv \mathrm{C} \underline{\mathrm{C}} \equiv \mathrm{C}), 65.9(\mathrm{C} \equiv \mathrm{CC} \equiv \underline{\mathrm{C}}), 63.7\left(\underline{\mathrm{CH}}_{2} \mathrm{OCO}\right), 61.9\left(\mathrm{O}^{\mathrm{C}} \mathrm{H}_{2}\right), 33.9\left(\underline{\mathrm{C}}\left(\mathrm{CH}_{3}\right)_{3}\right), 32.3$ $\left.\left(\mathrm{Ar} \underline{\underline{C}} \mathrm{H}_{2} \mathrm{Ar}\right)\right), 31.3\left(\left(\underline{\mathrm{C}} \mathrm{H}_{3}\right)_{3}\right), 27.8\left(\mathrm{C} \equiv \mathrm{CCH}_{2} \underline{\mathrm{C}} \mathrm{H}_{2}\right), 16.3\left(\mathrm{C} \equiv \mathrm{C}_{\mathrm{C}} \mathrm{H}_{2}\right)$. Anal. Calcd for $\mathrm{C}_{104} \mathrm{H}_{108} \mathrm{~N}_{4} \mathrm{O}_{12}: \mathrm{C}$, 77.78; H, 6.78; N, 3.49. Found: C, 77.39; H, 6.78; N 3.24. 